\title{
Polycaprolactone nanofibrous mesh reduces foreign body reaction and induces adipose flap expansion in tissue engineering chamber
}

This article was published in the following Dove Press journal:

International Journal of Nanomedicine

5 December 2016

Number of times this article has been viewed

\author{
Lin Luo ${ }^{1, *}$ \\ Yunfan $\mathrm{He}^{1,2, *}$ \\ Qiang Chang ${ }^{1,2}$ \\ Gan Xie' \\ Weiqing Zhan' \\ Xuecen Wang' \\ Tao Zhou' \\ Malcolm Xing ${ }^{2,3}$ \\ Feng Lu'
}

'Department of Plastic Surgery,

Nanfang Hospital, Southern Medical

University, Guangzhou, People's

Republic of China; 'Department of

Mechanical Engineering, Biochemistry

and Medical Genetics, University

of Manitoba, Winnipeg, Manitoba,

Canada, ${ }^{3}$ Children's Hospital

Research Institute of Manitoba,

Winnipeg, Manitoba, Canada

*These authors contributed equally to this work

\begin{abstract}
Tissue engineering chamber technique can be used to generate engineered adipose tissue, showing the potential for the reconstruction of soft tissue defects. However, the consequent foreign body reaction induced by the exogenous chamber implantation causes thick capsule formation on the surface of the adipose flap following capsule contracture, which may limit the internal tissue expansion. The nanotopographical property and architecture of nanofibrous scaffold may serve as a promising method for minimizing the foreign body reaction. Accordingly, electrospinning porous polycaprolactone (PCL) nanofibrous mesh, a biocompatible synthetic polymer, was attached to the internal surface of the chamber for the reducing local foreign body reaction. Adipose flap volume, level of inflammation, collagen quantification, capsule thickness, and adipose tissue-specific gene expression in chamber after implantation were evaluated at different time points. The in vivo study revealed that the engineered adipose flaps in the PCL group had a structure similar to that in the controls and normal adipose tissue structure but with a larger flap volume. Interleukin (IL)-1 $\beta$, IL-6, and transforming growth factor- $\beta$ expression decreased significantly in the PCL group compared with the control. Moreover, the control group had much more collagen deposition and thicker capsule than that observed in the PCL group. These results indicate that the unique nanotopographical effect of electrospinning PCL nanofiber can reduce foreign body reaction in a tissue engineering chamber, which maybe a promising new method for generating a larger volume of mature, vascularized, and stable adipose tissue.
\end{abstract}

Keywords: polycaprolactone nanofibrous mesh, topography, porous structure, adipose tissue regeneration, foreign body reaction

\section{Introduction}

The reconstruction of soft tissue defects following trauma (eg, severe burns), tumor resection (eg, mastectomy), or aging still presents a major challenge in plastic and reconstructive surgery. ${ }^{1,2}$ Current techniques used to reconstruct large soft tissue defects include artificial implants or autologous tissue transplantation, which are associated with certain limitations, including resorption and donor site morbidity. ${ }^{3-5}$

Tissue engineering offers an alternative to these suboptimal techniques, but vascularization is a major current limitation to the size, maintenance, and quality of engineered tissue. Based on Fick's diffusion theory, cells at a distance of more than $200 \mu \mathrm{m}$ from a blood vessel or capillary in vivo are either inactive or necrotic due to the limitations of nutrient diffusion..$^{6-8}$ In 2003, the tissue engineering chamber technique was introduced, which involves embedding a vascularized pedicled adipose flap in a chamber, making it possible to generate mature, vascularized, and 
transferable adipose tissue. ${ }^{9}$ In 2011 , the initial $5 \mathrm{~mL}$ of adipose flap successfully expanded to $56.5 \mathrm{~mL}$ using large volume chamber $(78.5 \mathrm{~mL})$ in pig model, providing clinically relevant volumes of tissue for soft tissue construction. ${ }^{10}$ Despite the advancement of tissue engineering chamber technique in recent years, ${ }^{11-13}$ the final maximum volume of the flap was still unable to fill the chamber thoroughly for unknown reasons. Efforts have been made to induce larger volume of the flap, including the usage of a larger volume chamber, exogenous growth factors, and extracellular matrix scaffolds. ${ }^{14-17}$ However, these methods are associated with the generation of toxic degradation products, risk of tumor formation, and high proportion of fibrous tissue in chambers. Thus, more feasible approaches are still needed to induce a larger volume adipose flap in tissue engineering chamber.

Various implant materials are used for the reconstruction of soft tissue defects as well as for aesthetic breast augmentation, with silicone being one of the most popular and accepted implantable biomaterials. Like all nonabsorbable implants, silicone may cause fibrous capsule formation of varying thickness. ${ }^{18,19}$ Myofibroblasts are contractile fibroblasts found in fibrous capsule, which provide a contractile force and decrease the surface area of the capsule, leading to the capsule contracture over time. ${ }^{20}$ Moreover, clinical study of capsular contractures after aesthetic breast augmentation revealed that the average tensile strength of the capsule was $44 \pm 38 \mathrm{~N},{ }^{21}$ and the intracapsular pressure correlated positively with the degree of capsule thickness. ${ }^{22}$ Chambers used in the tissue engineering chamber technique are mainly made of silicone, and the formation of the thick fibrous capsule on the adipose flap surface in silicone chambers has been frequently reported. ${ }^{10,11,13,23}$ Therefore, we speculated that the foreign body reaction induced by implanted silicone chambers would lead to capsule formation on the adipose flap surface and subsequent capsule contraction, which is similar to what happened with human silicone implants; this could be one of the major factors contributing to the limited final maximum volume of an engineered flap. Reducing the extent of foreign body reaction in silicone chamber would induce decreased fibrous capsule formation and reduce the degree of contraction on the adipose flap surface, which would ultimately lead to a larger volume of adipose flap.

Reducing the extent of foreign body reaction induced by subcutaneous implants remains a hot topic, with topographical modification being a research focus for minimizing the reaction in the past few years. Recent studies have indicated that material topographical features have a dramatic effect on foreign body reaction, ${ }^{24,25}$ and a porous structure tends to result in a moderate tissue response. ${ }^{26,27}$ Polycaprolactone
(PCL) nanofibrous scaffold, a biocompatible synthetic polymer with a relatively long degradable period, was reported to have a porous structure and was proven to be effective in suppressing foreign body reaction and fibrous capsule formation in vivo. ${ }^{28,29}$ With the decrease in intracapsular pressure, the neoformed tissue induced by a chamber undergoes further expansion.

To test our hypothesis, we fabricated porous PCL nanofibrous mesh using the electrospinning technique and attached it to the internal surface of a silicone chamber before implantation. Inflammation level, cell proliferation, collagen quantification, capsule thickness, and adipose tissue-specific gene expression in a chamber after implantation were evaluated at different time points.

\section{Materials and methods}

\section{PCL nanofibrous mesh preparation and} tensile test

PCL (Mw:80,000 g/mol, Sigma-Aldrich, St Louis, MO, USA, $10 \mathrm{wt} \%$ ) solution was prepared in dimethylformamide/ dichloromethane (Sigma-Aldrich 1:4, v/v) solvent mixture by gentle magnetic stirring at room temperature until completely dissolved. ${ }^{29,30}$ The solution was loaded into $10 \mathrm{~mL}$ Luer-lock syringe with 25 -gauge needle, and the infusion rate was regulated by a syringe pump (Harvard PHD syringe pump, Harvard Apparatus, Holliston, MA, USA). The infusion rate was set to $1 \mathrm{~mL} / \mathrm{h}$; the distance between the needle tip and steel mash collector was $18 \mathrm{~cm} .{ }^{31}$ High direct current voltage (Gamma High Voltage Research, Ormond Beach, FL, USA) was applied to the PCL solution. The electrospinning process was conducted at room temperature $\left(25^{\circ} \mathrm{C} \pm 1^{\circ} \mathrm{C}\right)$ and with the humidity maintained at $45 \%$. After electrospinning, the PCL nanofibrous mesh was torn off, and $1 \mathrm{~mm}$ thickness sheets were used at following in vivo test.

Mechanical test of PCL nanofibrous mesh was performed using Instron tensile tester (5965; Illinois Tool Works Inc., Glenview, IL, USA) at ambient environment. The sample was cut with scissors into $15 \times 10 \mathrm{~mm}^{2}$ rectangular shape and the thickness was measured with a vernier caliper, then the sample was placed between two crossheads, resulting in specimens dimension of $10 \times 10 \times 0.1 \mathrm{~mm}^{3}$, and the crosshead speed was set to $10 \mathrm{~mm} / \mathrm{min}$. Data, including stress (in $\mathrm{MPa}$ ) and elongation (\%), were automatically calculated by the Instron's Bluehill 2 software (Instron, High Wycombe, UK).

\section{Scanning electron microscopy evaluation of PCL nanofibrous mesh structure}

PCL nanofibrous mesh was cut into square samples $\left(1 \times 1 \mathrm{~cm}^{2}\right)$. The surface characteristics of the scaffolds were 
further investigated using the scanning electron microscope (Hitachi S-3000N; Hitachi Ltd., Tokyo, Japan). The mesh were mounted on carbon tape and coated with gold prior to imaging. An acceleration voltage of $20 \mathrm{kV}$ was used and images were observed using Canon digital camera (Canon Inc., Tokyo, Japan).

\section{Animals and tissue engineering chambers}

Adult male Sprague Dawley rats weighing 350-400 g were used for this study. All rats were housed on a 12-hour light/ dark schedule, and received a stock diet and water ad libitum. All experiments were performed under National Institutes of Health protocols and were approved by the Ethics Committee of Southern Medical University.

All operations were performed with general anesthesia (nembutal administered intraperitoneally at $60 \mathrm{mg} / \mathrm{kg}$ body weight). Cylindrical silicone chambers were manufactured by the clinical laboratory of Nanfang Hospital (Guangzhou, People's Republic of China). The perforated chambers were $15 \mathrm{~mm}$ long, with an internal diameter of $12 \mathrm{~mm}$ and a volume of $1.7 \mathrm{~mL}$.
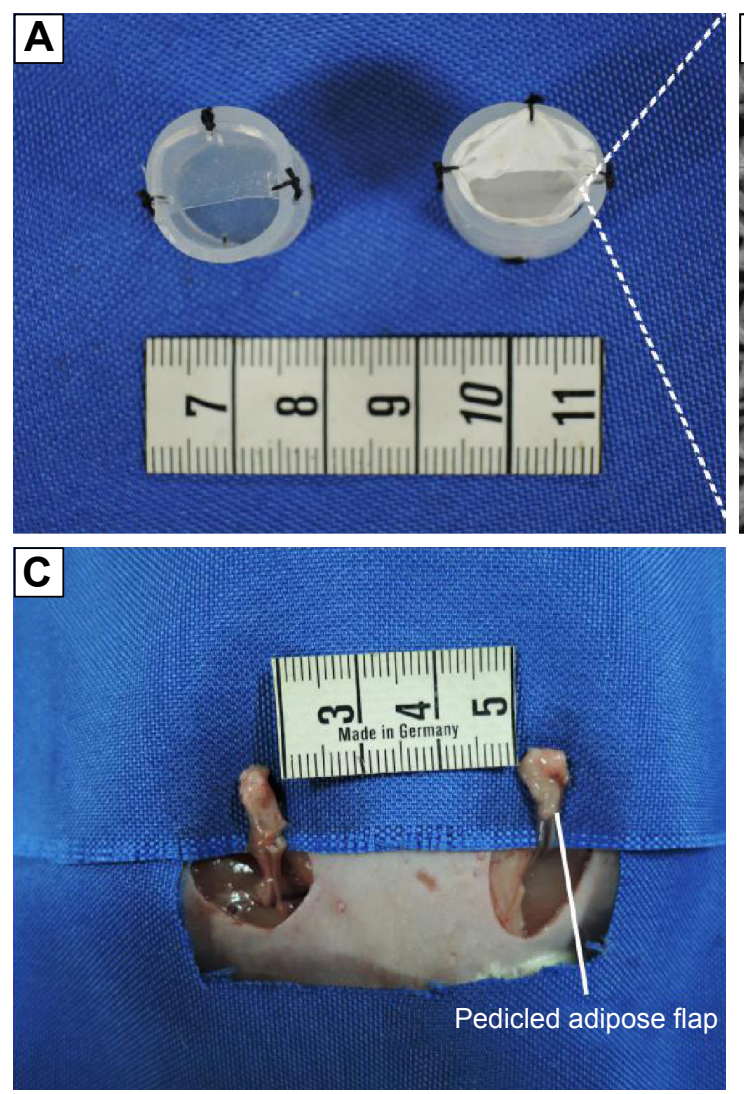

\section{Experimental design and surgical techniques}

Sprague Dawley rats $(n=66)$ were subdivided into experimental $(n=33)$ and control groups $(n=33)$. In the PCL group, the PCL nanofibrous mesh was attached to the internal surface of the chamber with 6-0 sutures (Figure 1A, right). Chambers without PCL nanofibrous mesh served as control (Figure 1A, left). A pedicled adipose flap based on the superficial inferior epigastric vessels of standardized volume was dissected in situ (Figure 1C).

Chambers were inserted into both groins of each rat (Figure 2A and B) as described in Dolderer et al's study. ${ }^{23}$ Briefly, the groins of rats were depilated with a hair remover, and the skin was then decontaminated with chlorhexidine and alcohol. A longitudinal incision was made in the groin to reveal the bilateral groin adipose pad. The pedicled adipose flap was embedded into the silicone chamber with a proper position to avoid the vasospasm, and then the chamber was anchored to underlying muscle with a 7-0 nylon microsuture. The wounds were closed with 4-0 sutures.
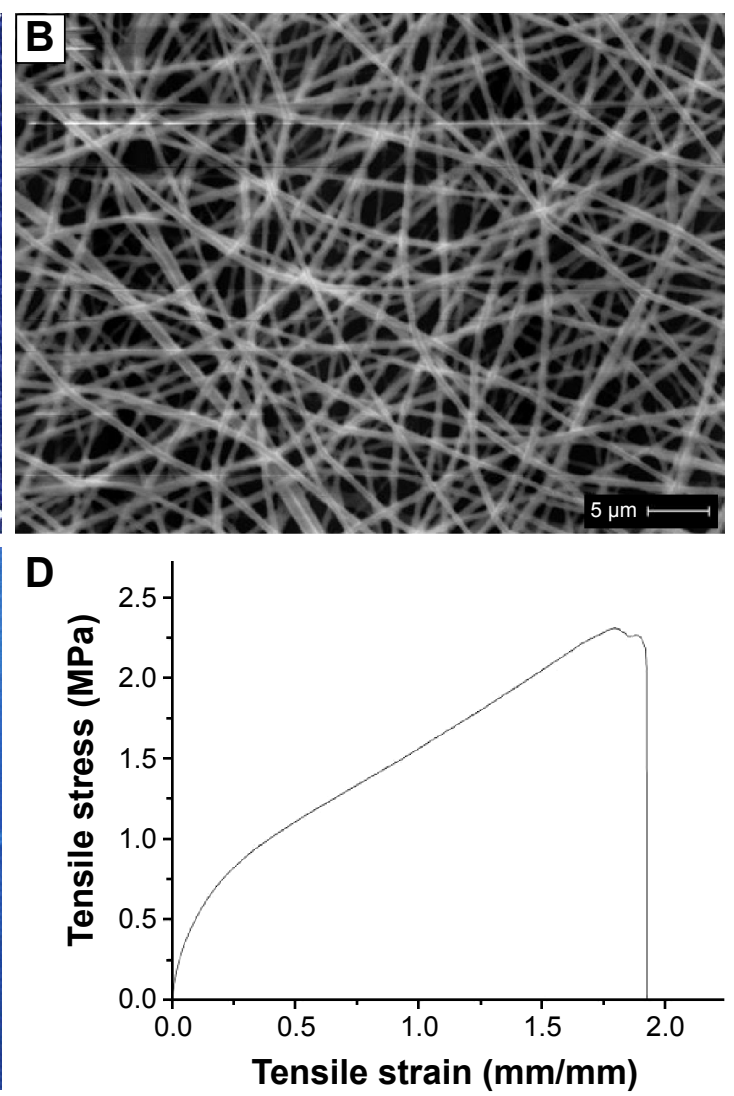

Figure I The rat chamber model and microstructure, stress-strain curve of PCL nanofibrous mesh.

Notes: (A) Cylindrical silicone chambers (left), PCL nanofibrous mesh was attached to the internal surface of the chamber (right). (B) SEM images of PCL nanofibrous mesh demonstrated the pore structure and nano-sized fibers. (C) A vascularized pedicled adipose flap based on the superficial inferior epigastric vessels of standardized dimensions was dissected in situ. (D) The stress-strain curve of PCL nanofibrous mesh.

Abbreviations: PCL, polycaprolactone; SEM, scanning electron microscopy. 


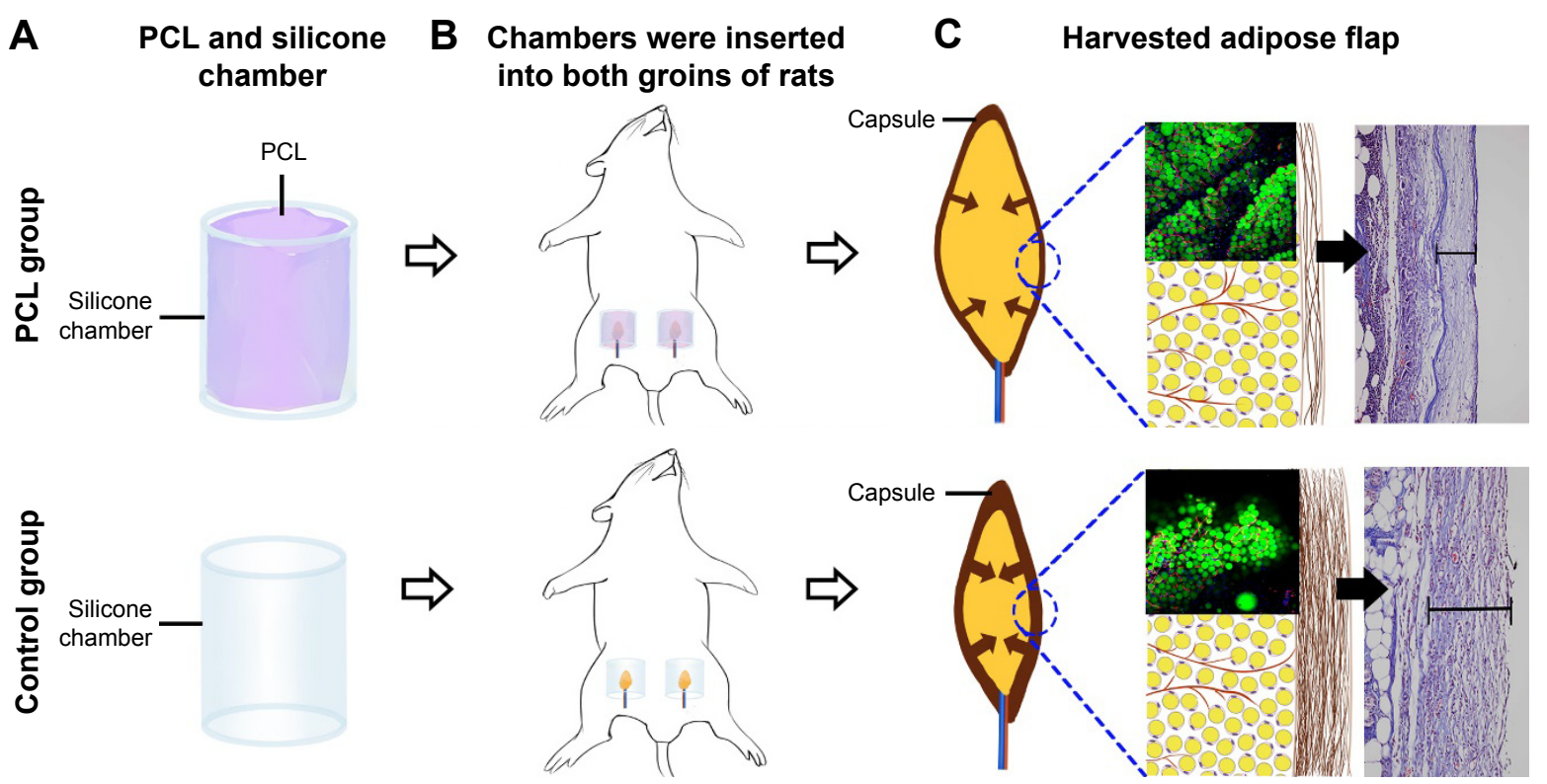

Figure 2 Animal model and harvested adipose flap.

Notes: (A) PCL nanofibrous mesh was attached to the internal surface of the cylindrical silicone chamber in the PCL group. Chambers without PCL nanofibrous mesh served as control. (B) Chambers embedded with a pedicled adipose flap were inserted into both groins of rats. (C) Adipose flap harvested at chamber removal at different time points.

Abbreviation: PCL, polycaprolactone.

\section{Tissue harvesting}

Rats were anesthetized at weeks $1,2,4,8$, and 12. Adipose flaps were harvested from chambers (Figure 2C), and their volumes were measured based on the volume of fluid displacement in normal saline. Each harvested sample was prepared for the following analyses. The anesthetized animals were killed by overdosed sodium pentobarbital injection (5 $\mathrm{mL}$ of a $20 \mathrm{mg} / \mathrm{mL}$ solution).

\section{Histological examination}

The tissue samples were fixed in $4 \%$ paraformaldehyde, dehydrated, and paraffin embedded for routine histological processing. Tissue blocks were serially sectioned $(4 \mu \mathrm{m}$ sections) along the longitudinal axis and stained using routine hematoxylin and eosin protocol, after which they were assessed under microscope and photographed with the digital camera (Olympus Corp., Tokyo, Japan).

\section{Whole-mount staining}

Harvested adipose samples were cut into $0.5-1 \mathrm{~mm}$ pieces after sacrifice and incubated with the following reagents for 30 minutes: BODIPY 558/565 (dilution 1:300; Molecular Probes, Eugene, OR, USA) to stain adipocytes, Alexa Fluor 488-conjugated isolectin GS-IB4 (dilution 1:200; Thermo Fisher Scientific) to stain endothelial cells, and Hoechst 33342 (dilution 1:500; Thermo Fisher Scientific) to stain nuclei. The samples were then washed and directly observed with a confocal microscope (FluoView ${ }^{\mathrm{TM}}$ FV1000, Olympus Corp., Tokyo, Japan).

\section{Immunohistochemistry for Ki67}

Sections were immunolabeled at different time points (weeks 4, 8, 12) from the samples of both groups.

The rabbit anti-rat Ki67 antibody (Abcam, Cambridge, MA, USA) was used to stain cells for proliferation. Briefly, paraffin sections were dewaxed and hydrated before immunohistochemical staining. Sections were washed in Tris-buffered saline and blocked with rabbit serum. Then, the sections were incubated with a primary antibody at $4^{\circ} \mathrm{C}$ overnight. After three wash steps, sections were incubated with the biotinylated secondary antibody (goat anti-rabbit IgG; Santa Cruz Biotechnology Inc., Dallas, CA, USA), diluted 1:200 in phosphate-buffered saline for 1 hour at room temperature. Slides were scored by two independent observers and photographed by Olympus DP71 digital camera (Tokyo, Japan).

\section{Collagen quantification}

For determination of collagen deposition in samples, adipose tissue obtained at all five time points from two groups were stained with Masson's trichrome. Briefly, sections were deparaffinized in xylene, rehydrated in graded ethanol, and postfixed in Bouin's fixative for 1 hour at $55^{\circ} \mathrm{C}$. The nuclei and 
collagen were sequentially stained with equal volumes of ferric chloride solution and alcoholic hematoxylin and trichrome solution, respectively. The total collagen content was reported as a percentage of the aniline blue staining divided by the total tissue area of the section using ImageJ software (National Institutes of Health, Bethesda, MD, America).

\section{Quantitative real-time polymerase chain reaction}

For real-time polymerase chain reaction (PCR) analysis, sample RNA was isolated from the chamber contents using Trizol and SYBR Green quantitative PCR SuperMix (Thermo Fisher Scientific), followed by reverse transcription. The complementary DNA samples were measured using customized TaqMan Array Plates (Thermo Fisher Scientific). Quantitative real-time PCR was carried out according to the TaqMan method $\left(50^{\circ} \mathrm{C}\right.$ for 2 minutes, $95^{\circ} \mathrm{C}$ for 10 minutes, and 40 cycles of $95^{\circ} \mathrm{C}$ for 15 seconds and $60^{\circ} \mathrm{C}$ for 1 minutes) with previously designed primers (Thermo Fisher Scientific) and was used for expression analysis. Relative expressions were calculated by cycle threshold method using $\beta$-actin as an endogenous reference gene. The mouse-specific primers used to amplify messenger RNA sequences were: Transforming growth factor beta 1 (TGF- $\beta 1$ ) forward 5'-TGCTTCAGCTCCACAGAGAA-3'; and reverse: 5'-TGGTTGTAGAGGGCAAGGAC-3'; Peroxisome proliferator-activated receptor gamma (PPAR $\gamma$ ) forward 5'-CTGGCCTCCCTGATGAATAA-3'; and reverse: 5'-GGCGGTCTCCACTGAGAATA-3'; IL-1 $\beta$ forward 5'-AGGCAGTGTCACTCATTGTGG-3'; and reverse: 5'-TAGCAGGTCGTCATCATCCC-3'; IL-6 forward 5'-GCCTTCTTGGGACTGATGTT-3'; and reverse: $5^{\prime}$-ACTGGTCTGTTGTGGGTGGT-3'; $\beta$-actin forward 5'-ACCCGCGAGTACAACCTTCTT-3'; and reverse: 5'-TATCGTCATCCATGGCGAACT-3'.

\section{Statistical analysis}

Data were expressed as mean \pm SD. results were analyzed with two-way analyses of variance (SPSS, version 21; IBM Corporation, Armonk, NY, USA). Furthermore, an independent Student's $t$-test of two groups at one time point was performed. A value of $P<0.05$ was considered statistically significant.

\section{Results}

\section{Analysis of PCL nanofibrous mesh pore size, structure, and mechanical property}

Scanning electron microscope was used to evaluate the size, structure, and diameters of the fibers within the film.
The PCL nanofibrous mesh exhibited a porous structure, and the diameter of the fibers was $550 \pm 69 \mathrm{~nm}$. The mean porosity was $89.4 \% \pm 2 \%$ (Figure $1 \mathrm{~B}$ ). The stress-strain curve was presented in Figure 1D. The mean value of Young's modulus was $2.4 \pm 0.4 \mathrm{MPa}$, and the average of elongation was $190 \% \pm 15 \%$. These values are consistent with previous reports of PCL nanofibrous mesh. ${ }^{32,33}$

\section{Morphometry and volume analysis at chamber removal}

Two animals died owing to infection. No significant inflammation or tissue necrosis was found at the silicone chamber implantation site. The PCL nanofibrous mesh was preserved perfectly and was attached to the internal surface of the silicone chamber in all animals in the PCL group. At week 4, after chamber removal, the PCL group consisted largely of a fragile, white, gel-like fibrin exudation surrounding the flap (Figure 3A) with small adipocytes detectable in the connective tissue (Figure 3B and C). However, by week 8 , new adipose tissue that had formed with newly formed blood vessels could be detected on the surface of fibrin exudation (Figure 3D and E). Similar features were observed in the constructs of the control group at 4 and 8 weeks, except that these constructs had a tougher tissue texture. By week 12, both groups developed vascularized and well-organized adipose tissue, with almost nonexistent fibrin exudation at the outermost layer (Figure 3G, H, and I).

Quantification of the flap volume revealed a gradual volume increase in both groups from week 0 to week 2 . The volume of the adipose flap increased from a baseline value of $0.100 \pm 0.060 \mathrm{~mL}$ to $0.140 \pm 0.012 \mathrm{~mL}$ in the PCL group and from $0.093 \pm 0.007 \mathrm{~mL}$ to $0.147 \pm 0.009 \mathrm{~mL}$ in the control group. The flap then showed a sharp increase to $0.357 \pm 0.029 \mathrm{~mL}$ and $0.250 \pm 0.023 \mathrm{~mL}$ at week 4 , and further increased to $0.440 \pm 0.026 \mathrm{~mL}$ and $0.330 \pm 0.023 \mathrm{~mL}$ at week 8 in the PCL and control groups, respectively. The flap volume of the PCL group was significantly larger than the control group as of week $4(P<0.05)$. The constructs volume in both groups appeared to reach full development by week 8 (Figure 4 ).

\section{Visualization of the tissue architecture in engineered adipose tissues}

Whole-mount staining was applied to visualize the tissue architecture and demonstrate the adipocyte and blood vessel interactions in the engineered tissue formed in the PCL group at week 12. For comparison, the control group was treated in the same manner. 



Figure 3 Engineered adipose flap in the PCL group chamber and hematoxylin and eosin-stained section at different time points.

Notes: The tissue of PCL group from week 4 consisted largely of a fragile, white, gel-like fibrin exudation surrounding the flap (A) with small adipocytes (inset) detectable in the connective tissue (B). By 8 weeks, new adipose tissue had formed (E) and small blood vessels could be detected on the surface of the fibrin exudation (D). Similar observations were made ain 4- and 8-week constructs of control groups (C, F), By week I2, both groups had developed vascularized and well-organized adipose tissue, with almost nonexistent fibrin exudation at the outermost layer (G, H, I). Scale bar $=100 \mu \mathrm{m}$.

Abbreviation: PCL, polycaprolactone.

At baseline, capillaries were observed between mature adipocytes in both groups. More mature and integrated structure of adipose tissue could be observed in the PCL group than the control group at week 12 (Figure 5).

\section{Angiogenesis and cell proliferation}

Proliferation marker Ki67 staining showed similar dynamic changes in Ki67+ cells in both groups. At week 4, abundant Ki67+ cells were scattered throughout the tissues, with

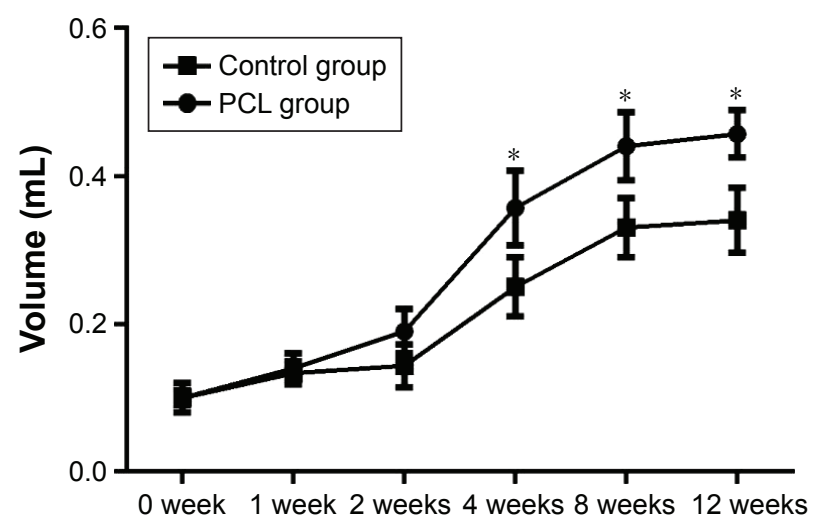

Figure 4 Volume changes in flaps at different time points.

Notes: There was a sharp volume increase (both groups) from week 2 to week 4 . The constructs volume in both groups appeared to have reached their full development by 8 weeks. Statistical analysis demonstrated that the flap volume of the PCL groups were significantly larger than the control group since week $4(* P<0.05)$.

Abbreviation: PCL, polycaprolactone. most of the Ki67+ cells located around vessels. However, the number of Ki67+ cells decreased sharply at week 8, and almost no Ki67+ cells were found at week 12 (Figure 6).

\section{Collagen deposition and changes in capsule thickness}

To demonstrate the collagen deposition in the tissue, adipose tissue harvested from both groups was stained with Masson's trichrome, and the areas of collagen staining (blue) were
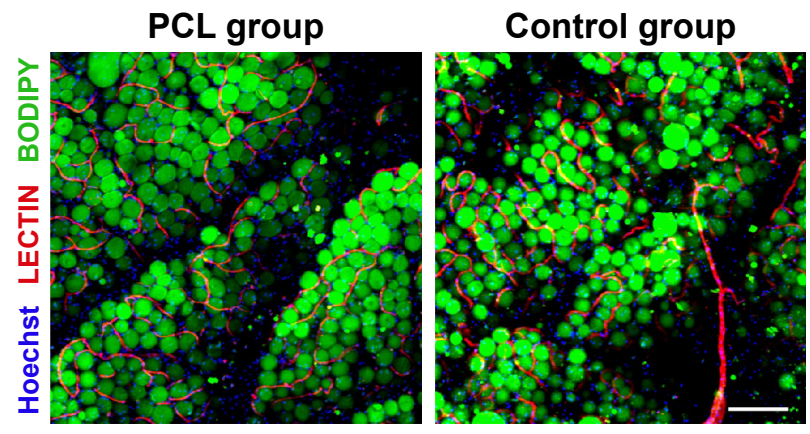

Figure 5 Visualization of engineered adipose tissue at week 12 by whole-mount staining.

Notes: Interaction of blood vessels and adipocytes in engineered adipose tissue (PCL group) was demonstrated by Lectin/BODIPY/Hoechst (red/green/blue) staining and compared with adipose tissue in control group. At baseline, capillaries or vessels ran between mature adipocytes in both groups, and adipose tissue in the $\mathrm{PCL}$ group displayed a more mature and integrated structure than the control group. Scale bar $=200 \mu \mathrm{m}$.

Abbreviation: $\mathrm{PCL}$, polycaprolactone. 

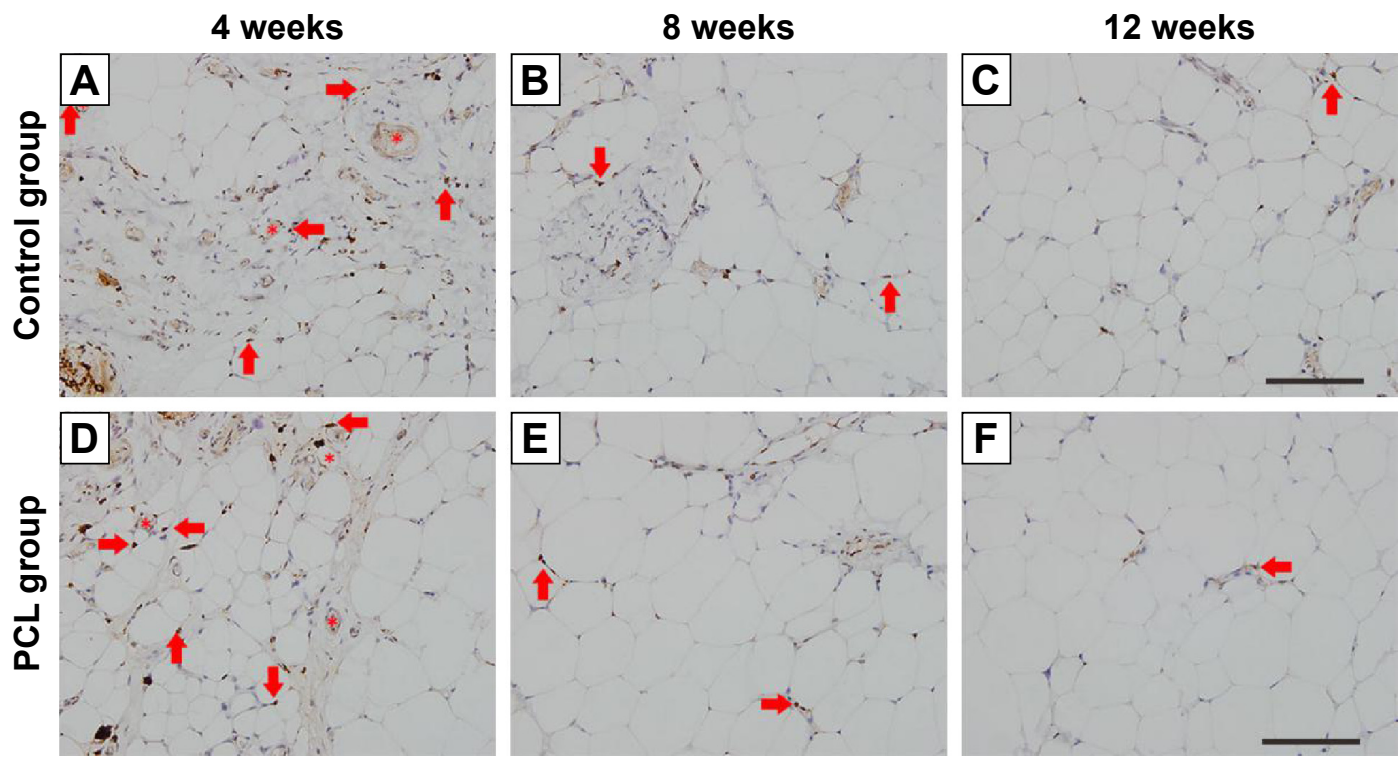

Figure 6 Ki67-stained section of the samples at different time points.

Notes: In both groups, most Ki67-positive cells (arrowhead) were located near blood vessels (star) at week 4 (A, D) and the number decreased at week 8 (B, E). Almost no Ki67-positive cells could be found at week 12 (C, F). Scale bar $=200 \mu \mathrm{m}$.

Abbreviation: PCL, polycaprolactone.

quantified. From week 1 to week 4, massive collagen deposition, limited to areas close to the pedicle, was found in both groups, and then the collagen was gradually replaced by newly formed small adipocytes by week 8 (Figure 7A-F). At week 12, there was a further decrease in the amount of collagen, and the remaining collagen was mainly observed near small blood vessels (Figure 7G and $\mathrm{H}$ ). Although the tissue structure was similar between weeks 1 and week 8 , quantification showed that the control groups maintained a higher collagen content throughout this period (Figure 7I).

Analysis of the aniline blue staining area was used to reflect the thickness of the flap capsule. At week 4, flaps in both groups were covered with a well-vascularized fibrous capsule. The thickness of the capsule reached its peak at week 8 , and then remained essentially unchanged from week 8 to week 12 (Figure 8A). Quantification showed that the adipose flap capsule of the control group was much thicker than that of the PCL group from week 4 to week 12 $(P<0.001)$ (Figure 8B).

\section{Gene expression of adipokine and other cytokines}

The messenger RNA expression level of PPAR $\gamma$, a master regulator of adipogenesis, ${ }^{11,34}$ was higher at week 4 in the PCL groups than the control groups (Figure 9A). The expression of activated TGF- $\beta$ showed a slight increase from week 1 to week 4 , and then decreased significantly from week 4 to 12 . However, the TGF- $\beta$ expression level in the PCL group was significantly lower than that in control groups from week 2 to week 4 (Figure 9B). The expression level of IL-1 $\beta$ was initially low but increased sharply from week 1 to week 2 , and then decreased continuously during the following weeks in both groups, with significantly higher relative expression in the control group than the PCL group from week 2 to week 4 (Figure 9C). Furthermore, IL-6 levels reached a peak at week 4, and then decreased slowly during the following weeks, with significantly higher relative expression in the control group than the PCL group at week 4 (Figure 9D). Interestingly, the level of inflammatory cytokines was consistent with the degree of collagen deposition in the tissue described earlier.

\section{Discussion}

Several studies have sought to generate vascularized and mature adipose tissue using the tissue engineering chamber technique. ${ }^{10,11,30,35}$ However, the size of engineered adipose tissue is limited owing to the formation of a thick fibrous capsule caused by the exogenous silicone chamber. In this study, we attached a biocompatible PCL nanofibrous mesh to the internal surface of the silicone chamber and observed a significant increase in total tissue volume after 2 weeks compared with the control.

In the present study, there were no marked structural and cellular differences observed in the engineered adipose tissue generated with the two methods, except for higher level of inflammation and thicker capsule formation in the control group than in the PCL group. The application of implants and devices using materials has thus far been limited due to 


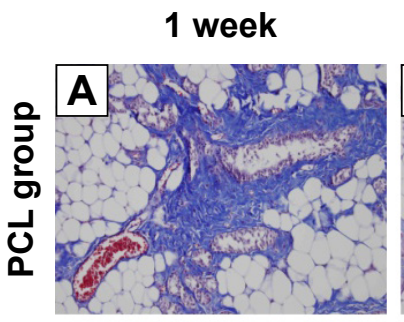

4 weeks
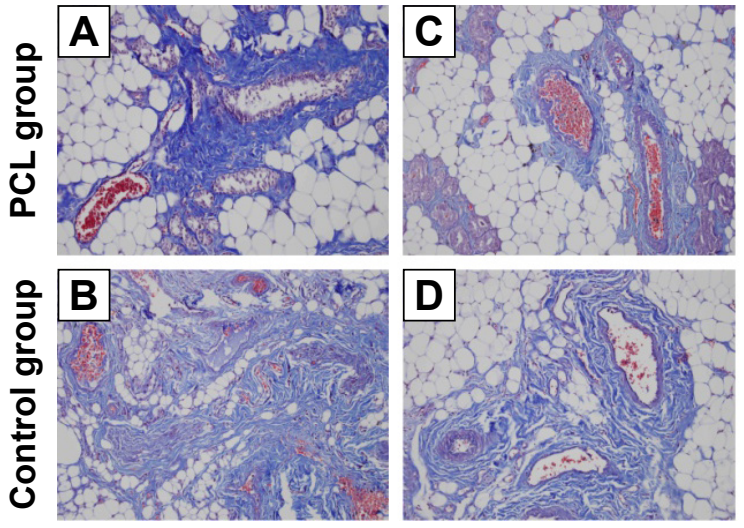

8 weeks
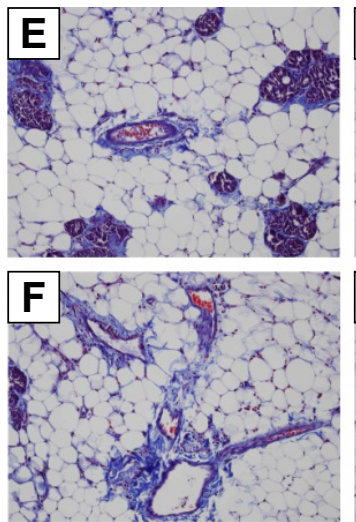

12 weeks
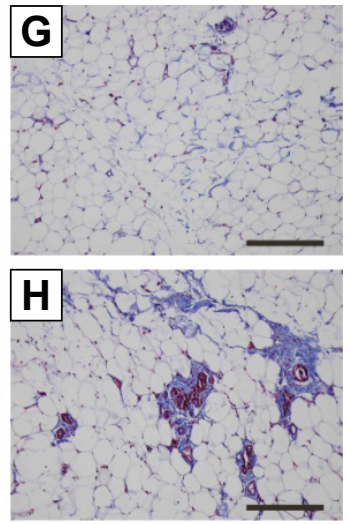

I

Area of positive staining

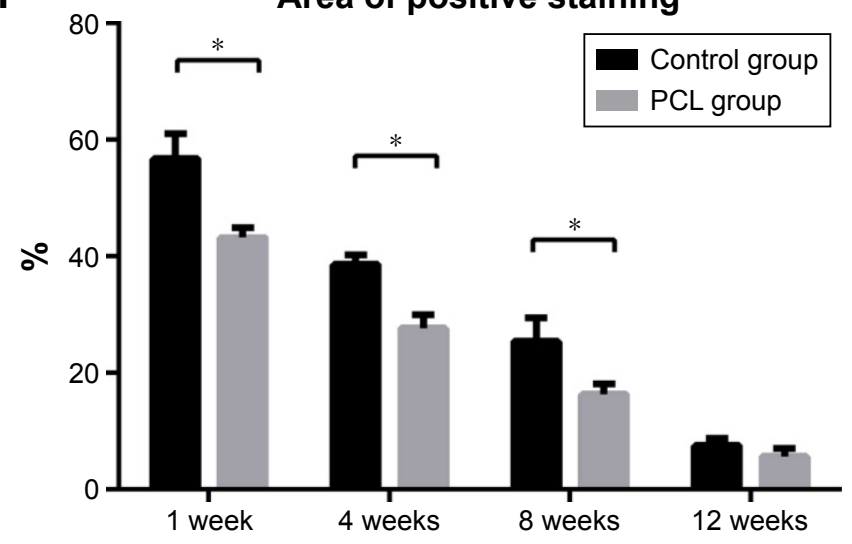

Figure 7 Collagen deposition at different times.

Notes: From weeks I to 4, massive collagen deposition, limited to the areas close to the pedicle, were found in both groups (A-D), then the collagens were gradually replaced by newly formed small adipocytes by week 8 (E, F). Statistical analysis demonstrated that the control groups still had higher collagen content between weeks I and 8 (I). At week 12, there was a further decrease in the amount of collagens, and the remaining collagens were observed mainly near small blood vessels (D, $\mathbf{H})$. All data were expressed as mean \pm standard deviation. $* P<0.05$ compared between $\mathrm{PCL}$ group and control group. Results are in response to an analysis of Student's $t$-test of two groups. Scale bar $=200 \mu \mathrm{m}$.

Abbreviation: $\mathrm{PCL}$, polycaprolactone.

the development of the foreign body reaction, an initial acute sterile inflammatory response, which subsequently overlaps with a chronic fibrotic response..$^{36-38}$ Initiation of the foreign body reaction involves nonspecific protein absorption and macrophage adhesion on the material surface, proceeding to giant cell formation, fibroblast activation, and collagen matrix deposition coupled with collagenous encapsulation. ${ }^{39}$

Foreign body encapsulation represents a chronic fibrotic response, and TGF- $\beta$ has been considered an important cytokine in this process. ${ }^{40-42}$ TGF- $\beta$ is rapidly released from platelets to initiate the healing process upon injury, and is subsequently produced by inflammatory cells (especially macrophages) in the wounds. ${ }^{40}$ In response to increased TGF- $\beta$, fibroblasts rapidly become activated and differentiate to myofibroblasts, which are characterized by a strong contractile capacity and high capacity for collagen synthesis. Under a normal wound-healing process, such a fibrotic response will ultimately minimize scarring and rebuild tissue integrity ${ }^{43}$ However, overexpression of TGF- $\beta$ often takes place during persistent chronic inflammation (eg, foreign body stimulation), leading to progressive capsule formation and subsequent capsule contraction..$^{19,44,45}$ There remains a high incidence of capsular contracture following cosmetic breast augmentation using silicone prosthesis. ${ }^{46,47}$ When it occurs, the implant is crumpled or fractured, with visible deformity of the implant and breast. ${ }^{48,49}$ In silicone chamber, there also exists a thick capsule around the engineered adipose flap, which has been considered to be a major reason limiting the further expansion of the flap mechanically at a later stage. In our study, lower TGF- $\beta$ expression at the early stage and reduced capsule formation at the late stage were found in the PCL group compared with the control group. This suggests that the PCL nanofibrous mesh in the chamber reduced capsule formation, which may be related to the decrease in TGF- $\beta$ expression. The finding is consistent with that reported by $\mathrm{Yu}$ et al, who found that a 

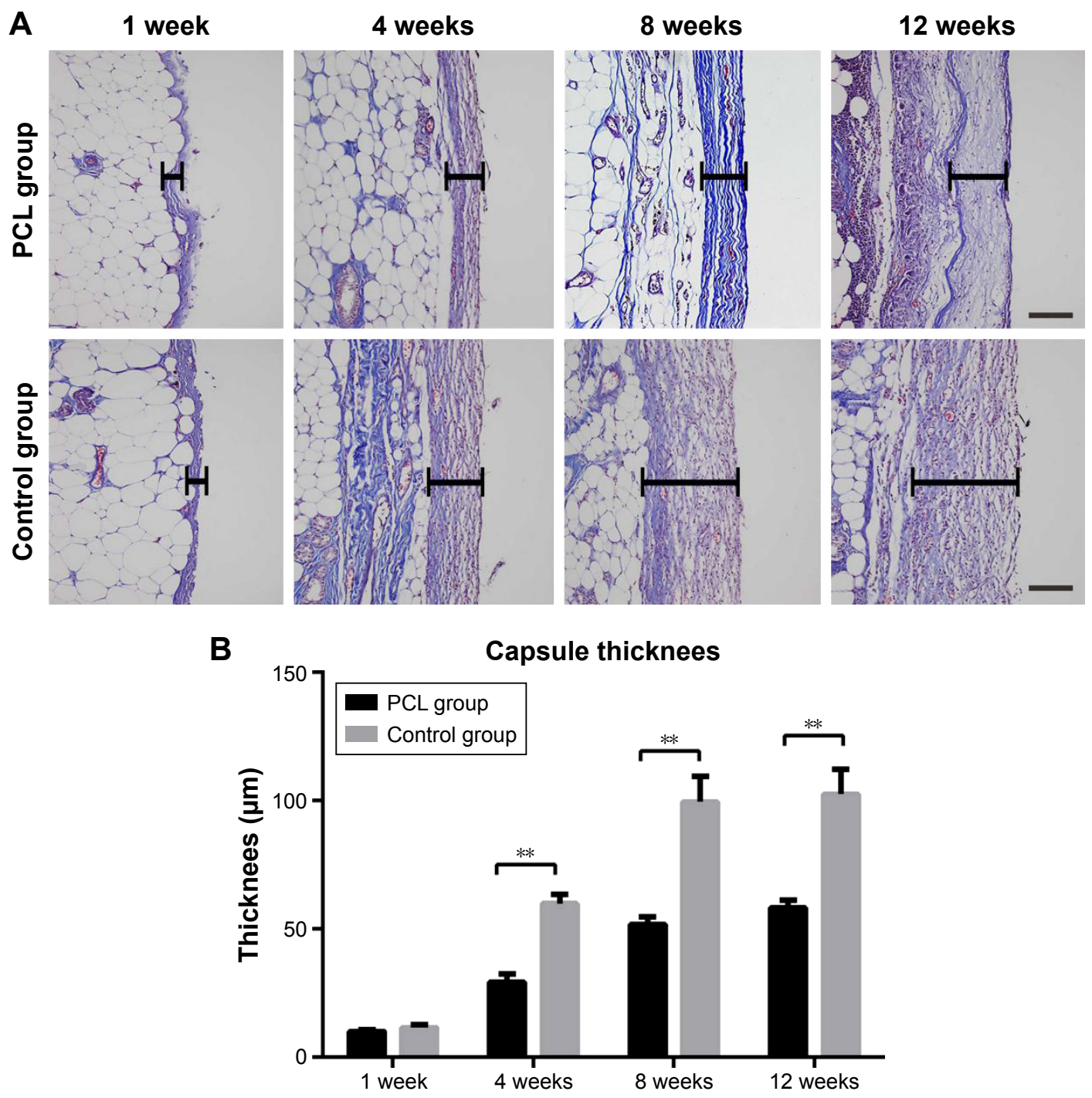

Figure 8 Changes in capsule thickness at different times.

Notes: The thickness of the capsule reached the peak at week 8, and then remained almost unchanged from weeks 8 to 12 (A). Statistical analysis demonstrated that the adipose flap capsule of the control group was much thicker than that of the PCL group from weeks 4 and 12 . All data were expressed as mean \pm standard deviation. $* * P<0.001$ compared between PCL group and control group. Results are in response to an analysis of Student's $t$-test of both groups $(\mathbf{B})$. Scale bar $=100 \mu \mathrm{m}$. Abbreviation: PCL, polycaprolactone.

lower concentration of TGF- $\beta$ in the tissue led to reduced capsular formation and contracture..$^{45}$ Moreover, it is also important to note that the massive collagen deposition observed in the control group at week 1 to week 4 may have indirectly contributed to capsular formation. However, the mechanism by which the PCL nanofibrous mesh might inhibit TGF- $\beta$ expression in the silicone chamber remains unclear. Recent studies indicated that the surface topographies of materials could considerably affect cellular behavior in the foreign body reaction. ${ }^{29,50}$ Porous structures tend to result in a moderate tissue response, and fibrous capsule formation on a porous surface is found to be thinner than that on a dense solid implant surface. ${ }^{26,51}$ After implantation of materials, macrophages and subsequent fibroblasts will migrate to the surfaces of the implants, releasing cytokines (eg, TGF- $\beta$ ) and depositing collagen to develop fibrous capsules. However, materials with large interfiber spacing result in a small fiber surface area for cell adhesion, leading to decreased cell density on the fiber surface. ${ }^{28}$ Therefore, the porous nano-sized surface geometry of a PCL nanofibrous mesh may lead to decreased cell (eg, macrophages and fibroblasts) adhesion compared with the planar, dense surface of silicone. This may further explain the decreased TGF- $\beta$ expression and reduced capsule thickness observed in the PCL group.

The severity of capsule formation and contracture also has a positive linear correlation with the degree of local inflammatory reactions. ${ }^{52}$ When the chambers are implanted, the surgical trauma and irritating exogenous materials initiate 
A

PPAR $\gamma$ level

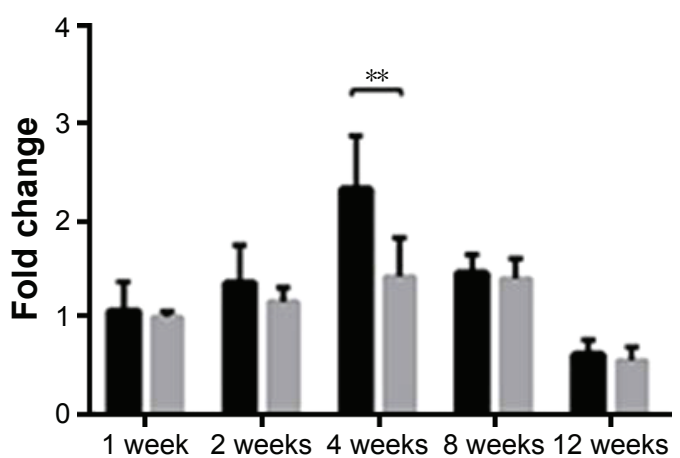

C

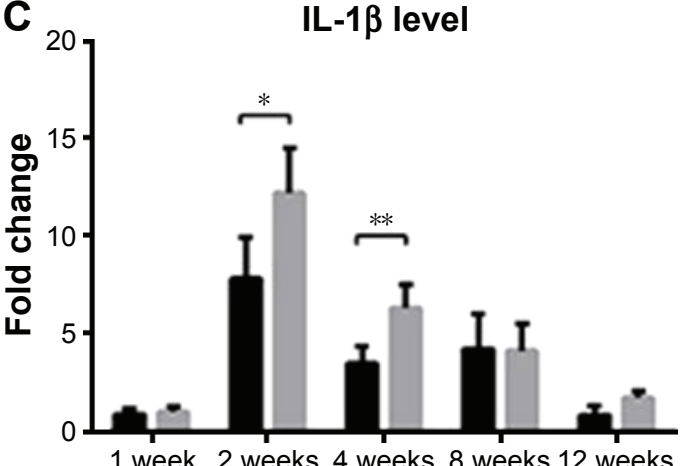

B

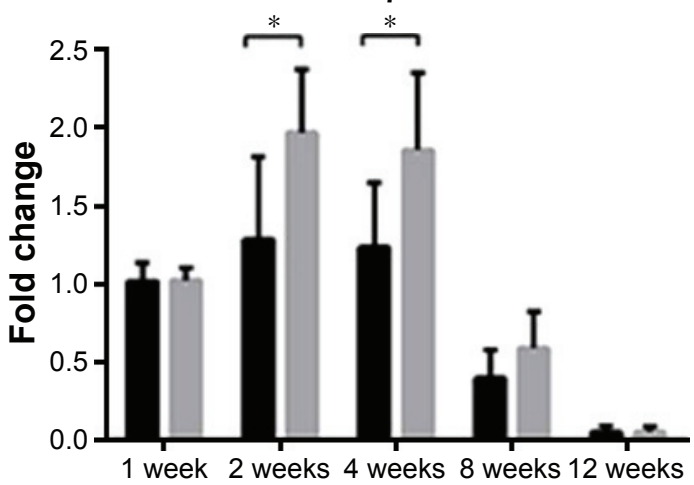

D

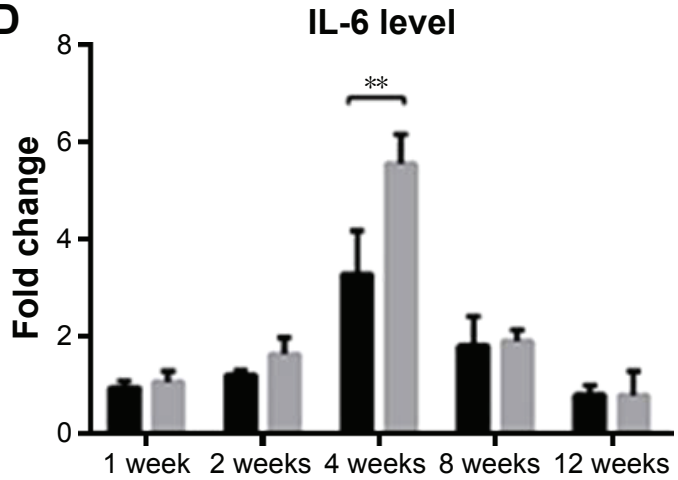

PCL group Control group

Figure 9 PCR characterization of gene expression of adipokine and other cytokines.

Notes: Real-time PCR analysis of the expression of adipogenic marker PPAR $\gamma(* * P<0.00 I)$, chronic fibrotic index TGF- $\beta(* P<0.05)$, inflammatory factors IL-I $\beta$ ( $* * P<0.00 I$, $* P<0.05)$, and IL-6 $(* * P<0.00 I, * P<0.05)$ in tissue from chamber. All data were expressed as mean \pm standard deviation. $* P<0.05$ and $* * P<0.00 \mathrm{I}$ compared between $\mathrm{PCL}$ group and control group. Results are in response to an analysis of Student's $t$-test of both groups.

Abbreviations: IL, interleukin; PPAR $\gamma$, peroxisome proliferator-activating receptor gamma; PCR, polymerase chain reaction; TGF, transforming growth factor.

an acute inflammatory response, leading to a local tissue reaction characterized by the secretion of a large amount of inflammatory factors such as monocyte chemotactic protein-1, IL-1 $\beta$, and IL- $6 .{ }^{53,54}$ Over the long term, the acute inflammatory reaction manifests as a chronic pattern because the chamber acts as a nonremovable injury stimulation.

In the present study, high levels of IL-1 and IL-6 were found in the control group at the early stage, and thick capsule formation was observed in the middle stage after implantation, which indicates that a severe inflammatory response contributes to increase capsule formation in chamber. Inflammation and angiogenesis are closely interrelated, ${ }^{55-57}$ and adipose angiogenesis is a key mediator in recruiting adipose precursor cells as well as the subsequent adipogenesis..$^{58,59}$ However, there was a reduced inflammatory level and larger final volume of adipose tissue in the PCL group compared with the control group in our study. Further researches revealed that adipogenesis is controlled by a complex interaction between positive and negative regulators. Besides inflammation, the extracellular microenvironment also has profound effects on cell functions, including proliferation, differentiation, and apoptosis. Low extracellular matrix stiffness and stretching (ie, decreased intracellular tension) promote adipogenesis, but compression impedes it. ${ }^{60-65}$ In our study, less collagen deposition and capsule formation were found in the PCL group at the early and middle stages compared with the control group, which indicated lower extracellular matrix stiffness and intracellular tension in the tissue of the PCL group in this period. This collective finding explains why there were more adipogenic gene $(P P A R \gamma)$ expression at week 4 and larger volume of adipose tissue in the following weeks in the PCL group.

Successful adipose flap expansion using the tissue engineering chamber technique provides a new option for the reconstruction of soft tissue defects. However, insufficient enlargement of the final tissue volume has been a major drawback with this method. We have demonstrated for the first time that the volume of vascularized adipose tissue in the silicone chamber can be further enlarged by reducing foreign body reaction and capsule formation using a biocompatible 
material without requiring complex exogenous angiogenic and adipogenic growth factors. In addition, there is no need for extracellular biological scaffold support with this method. Ongoing investigations are focusing on the specific mechanisms of capsule formation in the chamber. Obtaining a greater understanding of the relationship between adipogenesis and capsule formation has important implications for chamber design for the production of a larger volume of adipose tissue.

There has been a great advance in the development of biocompatible materials in recent years. In particular, topographical effect of biomaterial has been shown to minimize negative foreign body reaction in vivo. In this study, a porous nano-sized PCL electrospinning nanofibrous mesh was fabricated and applied in a silicone chamber to investigate the influence of the foreign body reaction on adipose tissue regeneration. Our findings suggest that PCL nanofibrous mesh can suppress extent of foreign body reaction in the chamber, leading to reduced capsule formation and a larger volume of the growing adipose flap. This knowledge could contribute new insight into the relationships between the foreign body reaction, capsule formation, and tissue regeneration.

\section{Acknowledgments}

This work was supported by National Nature Science Foundation of China (81471881, 81372083), Key Clinical Specialty Discipline Construction Program, Health Collaborative Innovation Major Projects of Guangzhou (7414275040815), Natural Science Foundation of Guangdong Province of China (2014A030310155), Entry Point Project of Guangdong Province of China (PY2014N036), Innovative Project of Guangdong Province of China (2014KQNCX046), and Administrator Foundation of Nanfang Hospital (2014B009).

\section{Disclosure}

The authors report no conflicts of interest in this work.

\section{References}

1. Bucky LP, Percec I. The science of autologous fat grafting: views on current and future approaches to neoadipogenesis. Aesthet Surg J. 2008;28(3):313-321.

2. Wan W, Zhang S, Ge L, et al. Layer-by-layer paper-stacking nanofibrous membranes to deliver adipose-derived stem cells for bone regeneration. Int J Nanomedicine. 2014;10:1273-1290.

3. Wan W, Li Q, Gao H, Ge L, Liu Y, Ouyang J, M Xing. BMSCs laden injectable amino-diethoxypropane modified alginate-chitosan hydrogel for hyaline cartilage reconstruction. $J$ Mater Chem B. 2014;3:1990-2005.
4. Chang Q, Gao H, Bu S, Zhong W, Lu F, Xing M. An injectable aldehyded 1-amino-3, 3-diethoxy-propane hyaluronic acid-chitosan hydrogels as carrier of adipose derived stem cells to enhance angiogenesis and promote skin regeneration. J Mater Chem B. 2015;3:4503-4513.

5. Jurkiewicz MJ, Nahai F. The use of free revascularized grafts in the amelioration of hemifacial atrophy. Plast Reconstr Surg. 1985;76(1):44-55.

6. Miller MJ, Patrick CJ. Tissue engineering. Clin Plast Surg. 2003; 30(1):91-103.

7. Patrick CJ, Chauvin PB, Hobley J, Reece GP. Preadipocyte seeded PLGA scaffolds for adipose tissue engineering. Tissue Eng. 1999;5(2): 139-151.

8. Zhang S, Xu K, Ge L, et al. A novel nano-silver coated and hydrogelimpregnated polyurethane nanofibrous mesh for ventral hernia repair. RSC Adv. 2016;6:90571-90578.

9. Hofer SO, Knight KM, Cooper-White JJ, et al. Increasing the volume of vascularized tissue formation in engineered constructs: an experimental study in rats. Plast Reconstr Surg. 2003;111(3):1186-1192; discussion 1193-1194.

10. Findlay MW, Dolderer JH, Trost N, et al. Tissue-engineered breast reconstruction. Plast Reconstr Surg. 2011;128(6):1206-1215.

11. Zhan W, Chang Q, Xiao X, et al. Self-synthesized extracellular matrix contributes to mature adipose tissue regeneration in a tissue engineering chamber. Wound Repair Regen. 2015;23(3):443-452.

12. Palmer JA, Abberton KM, Mitchell GM, Morrison WA. Macrophage phenotype in response to implanted synthetic scaffolds: an immunohistochemical study in the rat. Cells Tissues Organs. 2014;199(2-3): 169-183.

13. Ozcelik B, Blencowe A, Palmer J, et al. Highly porous and mechanically robust polyester poly(ethylene glycol) sponges as implantable scaffolds. Acta Biomater. 2014;10(6):2769-2780.

14. Xiao X, Yu L, Dong Z, et al. Adipose stem cell- laden injectable thermosensitive hydrogel reconstructing the depressed defect in rats: filler and scaffold. J Mater Chem B. 2015;3:5635-5644.

15. Ting ACH, Craft RO, Palmer JA, et al. The adipogenic potential of various extracellular matrices under the influence of an angiogenic growth factor combination in a mouse tissue engineering chamber. Acta Biomater. 2014;10(5):1907-1918.

16. Cronin KJ, Messina A, Knight KR, et al. New murine model of spontaneous autologous tissue engineering, combining an arteriovenous pedicle with matrix materials. Plast Reconstr Surg. 2004;113(1):260-269.

17. Vashi AV, Abberton KM, Thomas GP, et al. Adipose tissue engineering based on the controlled release of fibroblast growth factor- 2 in a collagen matrix. Tissue Eng. 2006;12(11):3035-3043.

18. Berry MG, Cucchiara V, Davies DM. Breast augmentation: part II adverse capsular contracture. J Plast Reconstr Aesthet Surg. 2010; 63(12):2098-2107.

19. Park S, Park M, Kim BH, et al. Acute suppression of TGF- $\beta$ with local, sustained release of tranilast against the formation of fibrous capsules around silicone implants. J Control Release. 2015;200:125-137.

20. Rudolph R, Abraham J, Vecchione T, Guber S, Woodward M. Myofibroblasts and free silicon around breast implants. Plast Reconstr Surg. 1978;62(2):185-196.

21. Hwang K, Sim HB, Huan F, Kim DJ. Myofibroblasts and capsular tissue tension in breast capsular contracture. Aesthetic Plast Surg. 2010;34(6):716-721.

22. Siggelkow W, Faridi A, Spiritus K, Klinge U, Rath W, Klosterhalfen B. Histological analysis of silicone breast implant capsules and correlation with capsular contracture. Biomaterials. 2003;24(6):1101-1109.

23. Dolderer JH, Abberton KM, Thompson EW, et al. Spontaneous large volume adipose tissue generation from a vascularized pedicled fat flap inside a chamber space. Tissue Eng. 2007;13(4):673-681.

24. Guillemette MD, Cui B, Roy E, et al. Surface topography induces 3D self-orientation of cells and extracellular matrix resulting in improved tissue function. Integr Biol (Camb). 2009;1(2):196-204.

25. Schulte VA, Diez M, Moller M, Lensen MC. Surface topography induces fibroblast adhesion on intrinsically nonadhesive poly (ethylene glycol) substrates. Biomacromolecules. 2009;10(10):2795-2801. 
26. Ward WK, Slobodzian EP, Tiekotter KL, Wood MD. The effect of microgeometry, implant thickness and polyurethane chemistry on the foreign body response to subcutaneous implants. Biomaterials. 2002; 23(21):4185-4192.

27. Vallejo-Heligon SG, Klitzman B, Reichert WM. Characterization of porous, dexamethasone-releasing polyurethane coatings for glucose sensors. Acta Biomater. 2014;10(11):4629-4638.

28. Cao H, McHugh K, Chew SY, Anderson JM. The topographical effect of electrospun nanofibrous scaffolds on the in vivo and in vitro foreign body reaction. J Biomed Mater Res A. 2010;93(3):1151-1159.

29. Chen S, Jones JA, Xu Y, Low HY, Anderson JM, Leong KW. Characterization of topographical effects on macrophage behavior in a foreign body response model. Biomaterials. 2010;31(13):3479-3491.

30. Li Q, Ge L,Wan W, et al. Magnetically Guided Fabrication of Multilayered Iron Oxide/Polycaprolactone/Gelatin Nanofibrous Structures for Tissue Engineering and Theranostic Application. Tissue Eng Part C Methods. 2015;21(10):1015-1024.

31. Jiang J, Wan W, Ge L, Bu S, Zhong W, Xing M. Mussel-inspired nanofibrous sheet for suture-less stomach incision surgery. Chem Commun. 2015;51:8695-8698.

32. Thomas V, Jose MV, Chowdhury S, Sullivan JF, Dean DR, Vohra YK. Mechano-morphological studies of aligned nanofibrous scaffolds of polycaprolactone fabricated by electrospinning. J Biomater Sci Polym Ed. 2006;17(9):969-984.

33. Croisier F, Duwez AS, Jerome C, et al. Mechanical testing of electrospun PCL fibers. Acta Biomater. 2012;8(1):218-224.

34. Lefterova MI, Lazar MA. New developments in adipogenesis. Trends Endocrinol Metab. 2009;20(3):107-114.

35. Doldere JH, Thompson EW, Slavin J, et al. Long-term stability of adipose tissue generated from a vascularized pedicled fat flap inside a chamber. Plast Reconstr Surg. 2011;127(6):2283-2292.

36. Onuki Y, Bhardwaj U, Papadimitrakopoulos F, Burgess DJ. A review of the biocompatibility of implantable devices: current challenges to overcome foreign body response. J Diabetes Sci Technol. 2008;2(6): 1003-1015

37. Kyriakides TR, Foster MJ, Keeney GE, et al. The CC chemokine ligand, CCL2/MCP1, participates in macrophage fusion and foreign body giant cell formation. Am J Pathol. 2004;165(6):2157-2166.

38. Malik AF, Hoque R, Ouyang X, et al. Inflammasome components Asc and caspase-1 mediate biomaterial-induced inflammation and foreign body response. Proc Natl Acad Sci U S A. 2011;108(50):20095-20100.

39. Wolfram D, Rainer C, Niederegger H, Piza H, Wick G. Cellular and molecular composition of fibrous capsules formed around silicone breast implants with special focus on local immune reactions. J Autoimmun. 2004;23(1):81-91.

40. Li AG, Quinn MJ, Siddiqui Y, et al. Elevation of transforming growth factor beta (TGFbeta) and its downstream mediators in subcutaneous foreign body capsule tissue. J Biomed Mater Res A. 2007; 82(2):498-508.

41. Mauviel A. Transforming growth factor-beta: a key mediator of fibrosis. Methods Mol Med. 2005;117:69-80.

42. Wynn TA. Fibrotic disease and the $\mathrm{T}(\mathrm{H}) 1 / \mathrm{T}(\mathrm{H}) 2$ paradigm. Nat Rev Immunol. 2004;4(8):583-594.

43. Massague J, Seoane J, Wotton D. Smad transcription factors. Genes Dev. 2005;19(23):2783-2810.

44. Ratner BD. Reducing capsular thickness and enhancing angiogenesis around implant drug release systems. J Control Release. 2002;78(1-3): 211-218.

45. Yu Q, Sheng L, Yang M, Zhu M, Huang X, Li Q. Tanshinon IIA injection accelerates tissue expansion by reducing the formation of the fibrous capsule. PLoS One. 2014;9(8):e105756.
46. Cunningham B. The Mentor Core Study on Silicone MemoryGel Breast Implants. Plast Reconstr Surg. 2007;120(7 Suppl 1):19S-29S; 30S-32S.

47. Spear SL, Murphy DK, Slicton A, Walker PS. Inamed silicone breast implant core study results at 6 years. Plast Reconstr Surg. 2007; 120(7 Suppl 1):8S-16S; 17S-18S.

48. Ben AM, Wu M, Trejo M, Atlan M. Morpho-elasticity of inflammatory fibrosis: the case of capsular contracture. J R Soc Interface. 2015; 12(111):20150343.

49. Headon H, Kasem A, Mokbel K. Capsular contracture after breast augmentation: an update for clinical practice. Arch Plast Surg. 2015; 42(5):532-543.

50. Paul NE, Skazik C, Harwardt M, et al. Topographical control of human macrophages by a regularly microstructured polyvinylidene fluoride surface. Biomaterials. 2008;29(30):4056-4064.

51. Hulbert SF, Morrison SJ, Klawitter JJ. Tissue reaction to three ceramics of porous and non-porous structures. J Biomed Mater Res. 1972; 6(5):347-374.

52. Poeppl N, Schreml S, Lichtenegger F, Lenich A, Eisenmann-Klein M, Prantl L. Does the surface structure of implants have an impact on the formation of a capsular contracture? Aesthetic Plast Surg. 2007;31(2): 133-139.

53. Peng Z, Dong Z, Chang Q, et al. Tissue engineering chamber promotes adipose tissue regeneration in adipose tissue engineering models through induced aseptic inflammation. Tissue Engineering Part C Methods. 2014;20(11):875-885.

54. Donizetti-Oliveira C, Semedo P, Burgos-Silva M, et al. Adipose tissuederived stem cell treatment prevents renal disease progression. Cell Transplant. 2012;21(8):1727-1741.

55. Lilja HE, Morrison WA, Han XL, et al. An adipoinductive role of inflammation in adipose tissue engineering: key factors in the early development of engineered soft tissues. Stem Cells Dev. 2013;22(10): $1602-1613$.

56. Hemmrich K, Thomas GP, Abberton KM, et al. Monocyte chemoattractant protein-1 and nitric oxide promote adipogenesis in a model that mimics obesity. Obesity (Silver Spring). 2007;15(12):2951-2957.

57. Thomas GP, Hemmrich K, Abberton KM, et al. Zymosan-induced inflammation stimulates neo-adipogenesis. Int J Obes (Lond). 2008;32(2): 239-248.

58. Tsuji T, Yamaguchi K, Kikuchi R, et al. Promotion of adipogenesis by an EP2 receptor agonist via stimulation of angiogenesis in pulmonary emphysema. Prostaglandins Other Lipid Mediat. 2014;112:9-15.

59. Li J, Qiao X, Yu M, et al. Secretory factors from rat adipose tissue explants promote adipogenesis and angiogenesis. Artif Organs. 2014; 38(2):E33-E45.

60. Assoian RK, Klein EA. Growth control by intracellular tension and extracellular stiffness. Trends Cell Biol. 2008;18(7):347-352.

61. Levy A, Enzer S, Shoham N, Zaretsky U, Gefen A. Large, but not small sustained tensile strains stimulate adipogenesis in culture. Ann Biomed Eng. 2012;40(5):1052-1060.

62. Pellegrinelli V, Heuvingh $\mathrm{J}$, du Roure $\mathrm{O}$, et al. Human adipocyte function is impacted by mechanical cues. J Pathol. 2014;233(2):183-195.

63. Young DA, Choi YS, Engler AJ, Christman KL. Stimulation of adipogenesis of adult adipose-derived stem cells using substrates that mimic the stiffness of adipose tissue. Biomaterials. 2013;34(34):8581-8588.

64. Hara Y, Wakino S, Tanabe Y, et al. Rho and rho-kinase activity in adipocytes contributes to a vicious cycle in obesity that may involve mechanical stretch. Sci Signal. 2011;4(157):ra3.

65. Shoham N, Gefen A. Mechanotransduction in adipocytes. J Biomech. 2012;45(1):1-8. 
International Journal of Nanomedicine

Dovepress

\section{Publish your work in this journal}

The International Journal of Nanomedicine is an international, peerreviewed journal focusing on the application of nanotechnology in diagnostics, therapeutics, and drug delivery systems throughou the biomedical field. This journal is indexed on PubMed Central, MedLine, CAS, SciSearch ${ }^{\circledR}$, Current Contents ${ }^{\circledR} /$ Clinical Medicine,
Journal Citation Reports/Science Edition, EMBase, Scopus and the Elsevier Bibliographic databases. The manuscript management system is completely online and includes a very quick and fair peer-review system, which is all easy to use. Visit http://www.dovepress.com/ testimonials.php to read real quotes from published authors.

Submit your manuscript here: http://www.dovepress.com/international-journal-of-nanomedicine-journal 